\title{
Majorana and the Infinite Component Wave Equations
}

\section{Roberto Casalbuoni}

Dipartimento di Fisica dell'Universita' di Firenze and Sezione INFN

Via G. Sansone 1, 50019 Sesto Fiorentino (FI), Italy

Galileo Galilei Institute for Theoretical Physics

L.go E. Fermi 2, 50125 Firenze, Italy

E-mail: casalbuoniefi.infn.it

\begin{abstract}
I review the paper of Majorana about relativistic particles with arbitrary spin written in 1932 . The main motivation for this papers was the dissatisfaction about the negative energy solutions of the Dirac equation. As such, the paper became immediately obsolete due to the almost contemporaneous discovery of the positron. However, for the first time, the unitary representations of the Lorentz group were introduced. Majorana considered two particular representations (named, after him, Majorana representations) which enjoy many interesting properties. A discussion about the reasons for its revival in the 60's is presented.
\end{abstract}

International Conference - Ettore Majorana's legacy and the Physics of the XXI century October 5-6 2006

University of Catania, Italy 


\section{Contents}

1. Introduction 2

2. The paper of Majorana about relativistic particles of arbitrary spin 3

$\begin{array}{lll}2.1 & \text { Four-vector operators } & 6\end{array}$

$\begin{array}{lll}2.2 & \text { Other interesting points discussed by Majorana } & 7\end{array}$

$\begin{array}{lll}\text { 3. Why the paper by Majorana was ignored? } & 8\end{array}$

4. The new interest in the 60's for the infinite component wave equations 9

$\begin{array}{llr}4.1 \text { Dynamical groups } & 9\end{array}$

$\begin{array}{lll}4.2 \text { Current algebra } & 10\end{array}$

5. The CPT theorem 12

$\begin{array}{lll}\text { 6. Conclusions } & 13\end{array}$

\section{Introduction}

In 1932 Ettore Majorana published a paper, in italian, by the title "Relativistic theory of particles with arbitrary angular momentum" [1]. As it is well known the physical interpretation of the Dirac equation [2] was rather problematic due the existence of negative energy solutions. In 1931 Dirac proposed a solution in terms of the hole-theory [3] introducing a new kind of particles with the same mass of the electrons and opposite charge, the positrons. The positron was discovered by Anderson [4] at the end of 1931, and the paper with the first picture of a positron appeared at the very beginning of 1932. It is not clear when Majorana wrote this paper (probably during the summer, according to Amaldi) and in which month of 1932 the paper appeared in Il Nuovo Cimento. However it seems that the news of the discovery of the positron arrived in Rome only around the end of 1932. So when Majorana conceived his paper the problem of the negative energy states was still in his mind. Therefore the aim of the paper was to construct a Dirac-like equation with only positive energy solutions. Majorana found that this is indeed possible, but that it is necessary that the wave function transforms under unitary representations (UR) of the homogeneous Lorentz group. These representations are infinite dimensional, as he discovered. The UR's were completely unknown at that time, not only among physicists but also among mathematicians. Majorana showed here his great mathematical ability and his mastering of group theory finding out two simple unitary representations for the wave function. I will discuss some of the reason why his paper was ignored at the time it was published, although it would have been of great interest from the point of view of group theory. Majorana's paper was recovered from the general ignorance 
thanks to Fradkin in 1966 that, stimulated by Amaldi whereas he was visiting Rome, published a comment to the Majorana's paper [5]. There were several reasons why this paper was of interest to physicists in that period. I will mention the incoming use of dynamical groups and the problem of saturating the algebra of currents at $p=\infty$ with a set of single particle states. I will discuss why the equations of Majorana type, involving unitary representations of the Lorentz group, were of interest for the previous topics. Another interesting point of the infinite component wave equations is that the CPT theorem does not generally hold. In fact the proof of the theorem in relativistic local field theories is valid only under the assumption that it is possible to perform an analytical continuation in the parameters of the Lorentz group. This condition is satisfied in the case of finite dimensional representations of the Lorentz group but it is not for UR's. I will discuss briefly this point.

\section{The paper of Majorana about relativistic particles of arbitrary spin}

In the paper of Majorana the following linear wave equation of the Dirac type was introduced:

$$
(E+\vec{\alpha} \cdot \vec{p}-\beta M) \psi=0
$$

Since Majorana wanted to avoid negative energy solutions, he required $\beta$ to be a positive definite operator. The other important point was that he did not require the validity of the Klein-Gordon equation, that is he did not ask for a single mass value associated to the wave function. As a consequence of the positivity of the operator $\beta$ it follows that the wave function must transform according to a UR of the Lorentz group. The argument of Majorana is very simple and it is based on writing down an action from which to derive the wave equation in a variational way. The action is

$$
\int d^{4} x \psi^{\dagger}(E+\vec{\alpha} \cdot \vec{p}-\beta M) \psi
$$

Since $\beta$ is required to be positive definite one can redefine the wave function according to

$$
\tilde{\psi}=\beta^{1 / 2} \psi
$$

The action becomes

$$
\int d^{4} x \tilde{\psi}^{\dagger}\left(\Gamma_{0} E+\vec{\Gamma} \cdot \vec{p}-M\right) \tilde{\psi}
$$

where

$$
\Gamma_{0}=\beta^{-1}, \quad \vec{\Gamma}=\beta^{-1 / 2} \vec{\alpha} \beta^{-1 / 2}
$$

From this one gets the wave equation

$$
\left(\Gamma_{\mu} p^{\mu}-M\right) \tilde{\psi}=0, \quad \Gamma_{\mu}=\left(\Gamma_{0}, \vec{\Gamma}\right), \quad p^{\mu}=(E, \vec{p})
$$

Since the action must be Lorentz invariant it follow that the same must be true for $\tilde{\psi}^{\dagger} \tilde{\psi}$. Therefore, under a Lorentz transformation $\tilde{\psi}$ must transform as a UR of the Lorentz group

$$
\tilde{\psi}^{\prime}=S \tilde{\psi}, \quad S^{\dagger} S=1
$$


The next step made by Majorana was to evaluate the commutation relations for the generators of the Lorentz group, which in modern notations read

$$
\left[J_{i}, J_{j}\right]=i \varepsilon_{i j k} J_{k}, \quad\left[J_{i}, N_{j}\right]=i \varepsilon_{i j k} N_{k}, \quad\left[N_{i}, N_{j}\right]=-i \varepsilon_{i j k} J_{k}
$$

where $\vec{J}$ are the generators of the rotation group and $\vec{N}$ the boost generators. The relation with the covariant generators $J_{\mu \nu}$ is:

$$
J_{k}=\frac{1}{2} \varepsilon_{k i j} J_{i j}, \quad N_{k}=J_{k 0}
$$

Majorana was an expert in group theory. He had in his bookshelf the Weyl's book on Quantum Mechanics and group theory [6], as well as other books more mathematically oriented in the subject. In particular in Weyl's book one can find the calculation of the matrix elements in the angular momentum basis of the electric dipole operator. On the other hand, according to Amaldi who knew the way of working of Majorana it is very well possible that he did the calculation by himself.

Let me now pause for a while in the exam of the paper and let me discuss a bit what we know today about the irreducible UR's of the Lorentz group. First of all, in complete generality, since the boosts are vector operators, their action on an angular momentum basis, $|j, m\rangle$, can be written as

$$
\begin{aligned}
N_{+}|j, m\rangle & =C_{j}[(j-m)(j-m-1)]^{1 / 2}|j-1, m+1\rangle-A_{j}[(j-m)(j+m+1)]^{1 / 2}|j, m+1\rangle \\
& +C_{j+1}[(j+m+1)(j+m+2)]^{1 / 2}|j+1, m+1\rangle \\
N_{-}|j, m\rangle & =-C_{j}[(j+m)(j+m-1)]^{1 / 2}|j-1, m-1\rangle-A_{j}[(j+m)(j-m+1)]^{1 / 2}|j, m-1\rangle \\
& -C_{j+1}[(j-m+1)(j-m+2)]^{1 / 2}|j+1, m-1\rangle \\
N_{3}|j, m\rangle & =C_{j}[(j-m)(j+m)]^{1 / 2}|j-1, m\rangle-A_{j} m|j, m\rangle \\
& -C_{j+1}[(j+m+1)(j-m+1)]^{1 / 2}|j+1, m\rangle
\end{aligned}
$$

where

$$
A_{j}=\frac{i j_{0} j_{1}}{j(j+1)}, \quad C_{j}=\frac{i}{j}\left[\frac{\left(j^{2}-j_{0}^{2}\right)\left(j^{2}-j_{1}^{2}\right)}{4 j^{2}-1}\right]^{1 / 2}
$$

These matrix elements depend on the pair $\left(j_{0}, j_{1}\right)$. These numbers characterize the Casimir operators of the Lorentz group

$$
\begin{aligned}
& C_{1}=\frac{1}{2} J_{\mu v} J^{\mu v}=\vec{J}^{2}-\vec{N}^{2}=j_{0}^{2}+j_{1}^{2}-1 \\
& C_{2}=\frac{1}{4} \varepsilon_{\mu v \rho \sigma} J_{\mu v} J_{\rho \sigma}=2 \vec{J} \cdot \vec{N}=2 i j_{0} j_{1}
\end{aligned}
$$

where we have used the matrix elements of the Lorentz generators. We see that $\left(j_{0}, j_{1}\right)$ and $\left(-j_{0},-j_{1}\right)$ are equivalent representations, therefore we may choose $j_{0}$ to be positive. It turns out that $j_{0}$ is the minimum angular momentum in the representation $\left(C_{j_{0}}=0\right)$. For finite dimensional representations $j_{1}=j_{\max }+1\left(C_{j_{\max }+1}=0\right)$. On the other hand in the infinite dimensional case the spin content of the representation is $j_{0}, j_{0}+1, \cdots$. Notice also that, since under parity $C_{2}$ changes sign, we have

$$
P:\left(j_{0}, j_{1}\right) \Rightarrow\left(j_{0},-j_{1}\right)
$$

So far about a generic irreducible representation. The unitarity condition selects two series of representations: 
- Principal series: $j_{0}$ integer or half-integer, $j_{1}$ pure imaginary.

- Supplementary series: $j_{0}=0, j_{1}$ real with $\left|j_{1}\right|<1$.

Going back to the paper of Majorana, after the equations defining the commutation relations among the Lorentz group generators he wrote: "The simplest solutions [of the commutation relations] by means of hermitian operators is given by the following infinite matrices" and he writes down the matrix elements of the Lorentz generators that can be obtained from the equations (2.10) by the choice $\left(j_{0}=0, j_{1}=1 / 2\right)$ or $\left(j_{0}=1 / 2, j_{1}=0\right)$. These two representations, later named after him, correspond to $C_{1}=-3 / 4$ and $C_{2}=0$ and are parity invariant. Majorana did not spend any other word about this simplicity, so it is not clear what he meant by it. One possibility is that he refers to the fact that with this choice the matrix elements of the boosts are particularly simple, since the coupling $j \rightarrow j$ vanishes and the other two couplings $j \rightarrow j \pm 1$ are given by $i / 2$. Since in his paper Majorana mentioned the fact that for his two representations $C_{2}=0$, it is also possible that he realized that with his choice the theory is parity invariant, but he did not state it explicitly. Also, quite strangely, he did not mention the other invariant operator $C_{1}$.

At this point Majorana went on to the evaluation of the matrix elements of the four-vector operator $\Gamma_{\mu}$. He wrote the commutation relations with the Lorentz generators that, using again modern notations, read

$$
\left[J_{\mu v}, \Gamma_{\rho}\right]=i\left(\Gamma_{\mu} g_{v \rho}-\Gamma_{v} g_{\mu \rho}\right)
$$

Then, Majorana wrote directly the matrix elements of $\Gamma_{\mu}$. These can be obtained by the observation that once $\Gamma_{0}$ is known, the $\Gamma_{i}^{\prime} s$ can be evaluated from

$$
\Gamma_{i}=-i\left[N_{i}, \Gamma_{0}\right]
$$

Noticing that $\Gamma_{0}$ is a scalar under rotations

$$
\left\langle j^{\prime}, m^{\prime}\left|\Gamma_{0}\right| j, m\right\rangle=\left\langle j, m\left|\Gamma_{0}\right| j, m\right\rangle \boldsymbol{\delta}_{j j^{\prime}} \boldsymbol{\delta} m m^{\prime}
$$

and using

$$
\Gamma_{0}=\left[\left[N_{i}, \Gamma_{0}\right], N_{i}\right]
$$

he got $\Gamma_{0}$ up to a constant. Choosing the constant to be one:

$$
\left\langle j, m\left|\Gamma_{0}\right| j, m\right\rangle=j+1 / 2
$$

Going to the rest frame (for the moment being we are considering time-like solutions) Majorana found the mass spectrum

$$
M_{j}=\frac{M}{j+1 / 2}, \quad j=j_{0}, j_{0}+1, \cdots, \quad j_{0}=0 \text { or } 1 / 2
$$

By reading the Majorana paper there is no trace about the fact that in order to write down a relativistic equation involving a four-vector operator one has to require very stringent constraints on the representation chosen. As we shall see, the two Majorana representations are the only irreducible representations for which these constraints are satisfied. 


\subsection{Four-vector operators}

We take another pause in the analysis of Majorana's paper to study the problem of a relativistic wave equation of the Dirac (or Majorana) type involving a four-vector operator. The consistency of the wave equation

$$
p \cdot \Gamma \psi=M \psi
$$

requires that the representation to which $\psi$ belongs is contained in the tensor product of the fourvector representation with the representation itself. Symbolically

$$
\psi \subset \Gamma_{\mu} \otimes \psi
$$

In order to evaluate this tensor product, let me first consider the case of finite dimensional representations. Remember that the Lorentz group is isomorphic to $S U(2) \otimes S U(2)$ and correspondingly a finite-dimensional representation can be denoted by $\left(s_{1}, s_{2}\right)$ where $s_{1}$ and $s_{2}$ are the spin content of the two $S U(2)$ representations. The physical spin operator is the sum of the two spin of the two commuting groups $S U(2) \otimes S U(2)$. The relation with the notation $\left(j_{0}, j_{1}\right)$ is

$$
j_{0}=\left|s_{1}-s_{2}\right|, \quad j_{1}=\left(s_{1}+s_{2}+1\right) \operatorname{sign}\left(s_{1}-s_{2}\right)
$$

The spin content of a four-vector is 0 and 1 . Therefore we have

$$
\Gamma_{\mu} \in\left(j_{0}, j_{1}\right)=(0,2) \Rightarrow\left(s_{1}, s_{2}\right)=(1 / 2,1 / 2)
$$

To evaluate the direct product of two finite dimensional representations of the Lorentz group is trivial in the basis $\left(s_{1}, s_{2}\right)$, since we have only to combine separately the spin of the two representations of $S U(2)$. We get

$$
\begin{aligned}
\left(\frac{1}{2}, \frac{1}{2}\right) \otimes\left(s_{1}, s_{2}\right) & =\left(s_{1}+\frac{1}{2}, s_{2}+\frac{1}{2}\right) \oplus\left(s_{1}+\frac{1}{2}, s_{2}-\frac{1}{2}\right) \oplus \\
& \oplus\left(s_{1}-\frac{1}{2}, s_{2}+\frac{1}{2}\right) \oplus\left(s_{1}-\frac{1}{2}, s_{2}-\frac{1}{2}\right)
\end{aligned}
$$

Going back to the $\left(j_{0}, j_{1}\right)$ notations we obtain

$$
(0,2) \otimes\left(j_{0}, j_{1}\right)=\left(j_{0}, j_{1}+1\right) \oplus\left(j_{0}+1, j_{1}\right) \oplus\left(j_{0}-1, j_{1}\right) \oplus\left(j_{0}, j_{1}-1\right)
$$

One can show that this relation holds for any irreducible representation $\left(j_{0}, j_{1}\right)$. Since the condition in eq. (2.20) reads

$$
\pm\left(j_{0}, j_{1}\right) \subset(0,2) \otimes\left(j_{0}, j_{1}\right)
$$

we find easily that the only solutions to this condition are indeed the two Majorana representations

$$
\left(j_{0}, j_{1}\right)=(0,1 / 2) \text { or }(1 / 2,0)
$$

More solutions can be found by relaxing the condition that $\psi$ belongs to an irreducible representation. Consider the case of "coupled representations". This means that

$$
\psi \in\left(j_{0}, j_{1}\right) \oplus\left(j_{0}^{\prime}, j_{1}^{\prime}\right)
$$


such that

$$
\left(j_{0}, j_{1}\right) \subset(0,2) \otimes\left(j_{0}^{\prime}, j_{1}^{\prime}\right), \quad\left(j_{0}^{\prime}, j_{1}^{\prime}\right) \subset(0,2) \otimes\left(j_{0}, j_{1}\right)
$$

In this way we satisfy the constraint of eq. (2.20). For instance, the Dirac representation

$$
\left(j_{0}, j_{1}\right)=\left(\frac{1}{2}, \frac{3}{2}\right) \oplus\left(\frac{1}{2},-\frac{3}{2}\right) \approx\left(\frac{1}{2}, 0\right) \oplus\left(0, \frac{1}{2}\right)=\left(s_{1}, s_{2}\right)
$$

satisfies this condition. If we are in the case $M=0$ there is no such constraint on the wave equation

$$
p^{\mu} \Gamma_{\mu} \psi=0
$$

and we get, for example, the Weyl's equations for massless spinors $(0,1 / 2)$ and $(1 / 2,0)$. For coupled representation one gets the Majorana equation for massive neutral particles [7], by observing that under hermitian conjugation, in a finite dimensional representation $C_{2} \rightarrow-C_{2}$ and therefore $j_{1} \rightarrow-j_{1}$ or equivalently $\left(s_{1}, s_{2}\right) \rightarrow\left(s_{2}, s_{1}\right)$. In particular $(1 / 2,0)$ is equivalent to $(1 / 2,0)^{*}$. More precisely

$$
i \sigma_{2} \chi^{*} \in(0,1 / 2), \text { if } \chi \in(1 / 2,0)
$$

As a consequence, it is possible to define a 4-component Majorana spinor by putting together $\chi$ and $\chi^{*}$

$$
\psi_{M}=\left(\begin{array}{c}
\chi \\
i \sigma_{2} \chi *
\end{array}\right)
$$

This spinor satisfies the condition of (pseudo-) reality

$$
C \psi_{M}=\left(\begin{array}{cc}
0 & -i \sigma_{2} \\
-i \sigma_{2} & 0
\end{array}\right)\left(\begin{array}{c}
\chi \\
i \sigma_{2} \chi *
\end{array}\right)=\left(\begin{array}{c}
\chi * \\
-i \sigma_{2} \chi
\end{array}\right)=\psi_{M}^{*}
$$

An interesting question is if Majorana was aware of the constraints on the representation in order to write a linear wave equation and in the affirmative case if this helped him in finding out the equation for neutral massive particles beyond the infinite component wave equation.

\subsection{Other interesting points discussed by Majorana}

We have seen that the Majorana equation gives rise to a mass spectrum for particles with different mass and spin. At that time this was not very interesting, since the spectrum of known particles was very poor (essentially, $p, e, \gamma$ and perhaps $n$ ). Therefore the idea of Majorana was rather to get the formalism to find the wave function for a particle with given spin and mass. This is something that it is possible to obtain from the Majorana wave equation in the non relativistic limit. In fact he shows that if one takes a solution of the wave equation, $\psi_{s, m}$, with fixed spin $s$, and mass $M /(s+1 / 2)$, then the wave functions for particles with different spin are suppressed by orders of $v / c$. For instance

$$
\psi_{s-1, m} \approx \mathscr{O}\left(\frac{v}{c}\right), \quad \psi_{s-2, m} \approx \mathscr{O}\left(\frac{v^{2}}{c^{2}}\right), \quad \cdots
$$

The proof goes like the decoupling of the negative energy solutions of the Dirac equation in the limit $v / c \rightarrow 0$. 
Another point analyzed by Majorana was the existence of space-like solutions. For space-like momenta one goes to the special frame

$$
p^{\mu} \rightarrow\left(0,0,0, \tilde{p}^{3}\right), \quad p^{2}=-\left(\tilde{p}^{3}\right)^{2}
$$

The equation becomes

$$
\tilde{p}^{3} \Gamma_{3} \psi=M \psi
$$

It is possible to diagonalize simultaneously $\Gamma_{3}$ and $J_{3}$

$$
\Gamma_{3}|\sigma, m\rangle=\sigma|\sigma, m\rangle, \quad J_{3}|\sigma, m\rangle=m|\sigma, m\rangle
$$

with $\sigma \geq 0$. The eigenvalue of $\Gamma_{3}$ is connected with the Casimir of the little group $S U(1,1)$ of the space-like momentum. Therefore one gets a continuous of space-like solutions:

$$
p_{0}^{2}-\vec{p}^{2}=-\frac{M^{2}}{\sigma^{2}}<0
$$

The Majorana equation has also a continuous of light-like solutions, but we will skip their discussion since they do not appear in the paper by Majorana.

Majorana wave equation was rediscovered and generalized (often without reference nor to the original paper neither to the existence of space-like solutions), as for instance in refs. [8,9] or in the books by Gel'fand, Minlos and Shapiro [10] and Naimark [11].

\section{Why the paper by Majorana was ignored?}

I will list here some of the topics that most probably contributed to take this paper out of the mainstream of research at that time.

We have already discussed the fact that the positron was discovered the same year of the Majorana equation and, presumably, the paper lost soon its physical interest also to the Majorana's eyes.

The Majorana wave equation gives rise to a mass spectrum, whereas at his time only a very restricted number of particles were known. Therefore this point did not make the theory very attractive, and Majorana himself did not pay too much attention to it.

Group theory was not yet very popular among physicists. On the contrary Majorana was very interested in this subject since his graduation time. In fact in his notes a lot of space is dedicated to group theoretical calculations.

The paper was written in italian and on Il Nuovo Cimento. This journal was not widely read at that time. Furthermore the Science Abstract did not abstract from Nuovo Cimento until 1946. On the other hand it was abstracted from Physikalische Berichte and the abstract of the Majorana's paper was reviewed there [12]. Unfortunately the abstractor was not an expert on the subject and the novelties of the paper, as the first treatment of the unitary representations of the Lorentz group, were not underlined.

In 1939 Wigner [13] solved completely the problem of the unitary representations of the Poincare group, i.e. the inhomogeneous Lorentz group. As it is well known physics requires that the wave function transforms under a UR of the latter group. These are obtained by fixing a 
representation of the translation group, that is to say the four-momentum, $p^{\mu}$. Given $p^{\mu}$ one has to choose a UR of the corresponding little group $(S U(2), S U(1,1)$ or $E 2$ according to a time-like, space-like or light-like momentum). This means that it is not mandatory to make use of UR's of the Lorentz group. Indeed in the Dirac representation only the angular momentum part is unitary, whereas the boost transformations are not. In the 30's (and even later), before Wigner's contribution, the situation was rather confused. This has lead Streater [14], commenting his own article [15] about the problems of the infinite component wave equations to the following considerations about the Dirac equation:

I conjecture that Dirac had the mistaken belief that his (Dirac) equation did not give rise to a unitary representation of the inhomogeneous Lorentz group (the Poincare group), because the $4 \times 4$ matrices appearing in it were not unitary. He may have realised the great importance of unitary representations after Wigner's book, Group Theory with applications to atomic spectroscopy. It might be that the fear that his equation were badly wrong urged Dirac to invent, in about 1945, single-handedly, some irreducible unitary representations of the Lorentz group, a task thought to be too hard for mathematicians at the time. If so, it was all to no avail, as the unitarity of the representation (of the Poincaré group, as opposed to the Lorentz group acting on the spinors) given by the original Dirac equation was shown by Wigner (1939) and by Bargmann and Wigner (1947).

We have already noticed that the Majorana wave equation was later rediscovered, often without any reference to the original paper. However in 1966, Fradkin, after a suggestion by Amaldi published a paper in english [5] commenting the Majorana's paper. In fact at that time two lines of research pointed toward infinite component wave equations. These two topics were:

- Dynamical groups.

- The Gell-Mann's program of saturating the algebra of currents at $p=\infty$ in terms of single particle states.

I will discuss the reasons for this renewal of interest in the next Section. I should also mention that the possibility of getting a mass spectrum was rather interesting from the point of view of the Regge theory.

\section{The new interest in the 60's for the infinite component wave equations}

\subsection{Dynamical groups}

In the 60's the main roads to strong interactions were the analytical $S$-matrix and group theory. A very important result obtained by group theory was the discovery of the symmetry $S U(3)$ as a classification group for hadrons and its unification with the rotation group leading to the $S U(6)$ symmetry $[16,17,18]$. A justification of the success of this group was the idea that hadrons were composite objects. In this sense it was natural to try to learn something from the simplest known system, the hydrogen atom. The similarity of the problems leading to $S U(6)$ for hadrons and to $O(4)$ for the hydrogen atom, unifying the rotation group with an internal symmetry $(S U(3)$ for hadrons and the transformations generated by the Runge-Lenz vector for the $\mathrm{H}$-atom), was discussed in ref. [19]. In 1967, Barut and Kleinert [20] and Fronsdal [21] found that it was possible 
to enlarge the symmetry group $O(4)$ of the H-atom to a dynamical group $O(4,2)$ which included, among its generators, the electric dipole operator. The name of this approach, "dynamical groups" was due to the hope to be able to describe the interactions as generators of a group, as it happens for the electromagnetic interaction in the $\mathrm{H}$-atom. It is interesting that the previous authors were able to prove that the Schrödinger equation for the hydrogen, taking also into account the electromagnetic interaction, can be rewritten in the form of a non-relativistic infinite component wave equation of the Majorana type. The connection between composite systems and UR's of the Lorentz group was already known by Dirac [22] (see also the Streater's comment [14]) who discussed the UR of this group in terms of continuous variables (instead of using a discrete basis as in the Majorana's approach). Eventually this idea of Dirac was the basis of the bilocal field theory introduced by Yukawa $[23,24]$. On the basis of these considerations several authors discussed a series of infinite component relativistic wave equations [25, 26, 27]. However all these equations showed a number of diseaeses as:

- Presence of redundant solutions, as the space-like ones.

- Typically these equations violate the CPT theorem (as for the Majorana case where no negative energy solutions are present).

- The spin-statistics theorem does not generally hold [28, 29, 15].

As a consequence of these problems the program of dynamical groups died very rapidly.

\subsection{Current algebra}

Let me consider the matrix elements of a vector current (to fix the ideas I will take currents of $S U(3) \otimes S U(3))$ in the limit of external momenta going to infinity [30]. To this end let me define

$$
F_{i}(\vec{q})=\int d^{3} \vec{x} e^{i \vec{q} \cdot \vec{x}} j_{i}^{0}(\vec{x}, 0), \quad i \in S U(3) \otimes S U(3)
$$

The matrix elements of this operator at $p=\infty$ can be written as

$$
\lim _{p_{3}, p_{3}^{\prime} \rightarrow \infty}\left\langle\vec{p}^{\prime}, N\left|F_{i}(\vec{q})\right| \vec{p}, N\right\rangle=\delta^{3}\left(\vec{p}^{\prime}+\vec{q}-\vec{p}\right)\left(N^{\prime}\left|J_{i}\left(\underline{p}^{\prime}-\underline{p}\right)\right| N\right)
$$

where $\underline{p}=\left(p_{1}, p_{2}\right)$. In the previous equation the states $\left.\mid N\right)$ are fictitious states that, in the case of single particles, depend only on the quantum numbers $N$. The algebra of the operators $J_{i}(\underline{p})$ is

$$
\left[J_{i}(\underline{p}), J_{j}\left(\underline{p}^{\prime}\right)\right]=i f_{i j k} J_{k}\left(\underline{p}+\underline{p}^{\prime}\right), \quad J_{i}^{\dagger}(\underline{p})=J_{i}(-\underline{p})
$$

The idea was to look for representations of this algebra in the space of states of single particle [31]. As shown by Coester and Roepstoff [32], in the space of single particle states, this algebra has only infinite dimensional representations.

An obvious representation of this algebra is:

$$
\begin{gathered}
J_{i}(\underline{p})=\sum_{n} \frac{1}{2} \lambda_{i}^{(n)} e^{i \underline{p} \cdot \underline{x}^{(n)}}, \quad\left[x_{1}^{(n)}, x_{2}^{(m)}\right]=0 \\
n=1,2 \text { for mesons, } \quad n=1,2,3 \text { for baryons }
\end{gathered}
$$


Unfortunately $J_{i}$ arises as a limit of the fourth component of a four-vector operator and therefore there is a set of complicated conditions that it must satisfy, the so called angular conditions [33]. This can be easily understood considering the matrix element

$$
\left\langle\vec{p}^{\prime}, N^{\prime}\left|j^{\mu}\right| \vec{p}, N\right\rangle, \quad \text { with } j^{\prime}=0, j \geq 1
$$

It depends on 4 invariant form factors whereas, without requiring any other condition, $\left(N^{\prime}|J(\underline{p})| N\right)$ depends on $2 j+1$ form factors. As I said the angular conditions are very complicated and just to convince you I will write their infinitesimal form

$$
\left\{I,\left\{I,\left\{I, J_{i}(\underline{p})\right\}\right\}\right\}=\frac{1}{4}\left[M^{2},\left[M^{2},\left\{I, J_{i}(\underline{p})\right\}\right]\right]++\frac{1}{2}|\underline{p}|^{2}\left[M^{2},\left\{I, J_{i}(\underline{p}\}\right]+\frac{1}{4}|\underline{p}|^{4}\left\{I, J_{i}(\underline{p})\right\}\right.
$$

where

$$
\left\{I, J_{i}(\underline{p})\right\}=\frac{1}{2}\left[M^{2},\left[L_{3}, J_{i}(\underline{p})\right]\right]-\frac{1}{2}|\underline{p}|^{2}\left[L_{3}, J_{i}(\underline{p})\right]_{+}-\left[\underline{p} \cdot M \underline{L}, J_{i}(\underline{p})\right], \quad \underline{L}=\left(L_{1}, L_{2}\right)
$$

Furthermore

$$
\left[L_{3}, J_{i}(\underline{p})\right]=i \underline{p} \wedge \underline{\nabla}_{p} J_{i}(\underline{p}), \quad \underline{\nabla}_{p}=\left(\frac{\partial}{\partial p_{1}}, \frac{\partial}{\partial p_{2}}\right)
$$

When all the particles have the same mass, $M$, the exponential solution (see eq. (4.4)) satisfies all these conditions with a position operator given by

$$
\left(x_{1}, x_{2}\right)=\frac{1}{M}\left(F_{1}, F_{2}\right)=\frac{1}{M}\left(N_{1}+J_{2}, N_{2}-J_{1}\right)
$$

where $\left(F_{1}, F_{2}\right)$ are the $E 2$ generators (the little group of light-like momenta). Starting from the degenerate case it is possible to find an approximate solution through a perturbative expansion in the splitting mass term $[34,35]$.

It is easy to see that one can avoid the problem of solving the angular conditions by using an appropriate infinite component wave equation. This is done by the following steps:

- Introduce a wave function, $\psi(x)$ transforming according to some UR of the Lorentz group with an appropriate spin content.

- Write an invariant wave equation with the desired mass spectrum:

$$
D(x) \psi(x)=0
$$

However notice that the mass squared should not increase more than the angular momentum as shown in ref. [36] (an equation with a linear mass spectrum was proposed in [37]).

- Require that the wave equation is invariant under a specified internal symmetry group.

Using the last requirement one can construct an algebra of conserved currents satisfying automatically current algebra as a consequence of the canonical commutation relations among the fields.

Of course there are problems very similar to the ones listed for the case of dynamical groups, but the real problem here (and also in the other case) is the presence of the space-like solutions. Before discussing further this point I want to stress that, immediately after the formulation of the program of saturating the current algebra, a No-Go theorem was formulated by Grodsky and Streater [38]. These authors made the following assumptions: 
1. existence of covariant wave functions,

2. reasonable mass spectrum: only time-like solutions with finite degeneracy on each mass shell,

3. the representation of the wave function should contain at least one finite-dimensional representation of the Lorentz group and a four-vector operator should exist,

4. the solutions of the wave equation form a complete set,

and proved that each mass-shell must be infinitely degenerate. Therefore the set of solutions satisfying the previous assumptions is void. On the other hand the theorem does not hold if the wave equation has space-like solutions. There could be a way out if the space-like solutions would decouple from the time-like ones, or said in other words, if the space-like solutions would not contribute to the completeness relation. It is possible to show that in these theories the decoupling of the space-like solutions is related to the possibility of proving the CPT theorem (see the discussion in ref. [39]). We shall see, in the next Section, that it is not possible to prove the CPT theorem for UR's and therefore the decoupling of space-like solutions cannot occur.

\section{The CPT theorem}

Consider a wave equation of the Dirac or Majorana type. The wave operator will be CPT invariant if it possible to define the following operation

$$
p^{\mu} \rightarrow-p^{\mu}, \quad \Gamma_{\mu} \rightarrow-\Gamma_{\mu}
$$

The transformation of $\Gamma_{\mu}$ can be obtained through a rotation of $\pi$ along the third axis followed by a boost along the same direction by an imaginary boost parameter $\xi=i \pi$ :

$$
R_{3}(\pi) \Gamma_{1,2} R_{3}(\pi)^{-1}=-\Gamma_{1,2}, \quad B_{3}(i \pi) \Gamma_{0,3} B_{3}(i \pi)^{-1}=-\Gamma_{0,3}
$$

In the case of the Dirac equation:

$$
B_{3}(i \pi) R_{3}(\pi)=\left(\gamma_{0} \gamma_{3}\right)\left(\gamma_{1} \gamma_{2}\right)=\gamma_{0} \gamma_{1} \gamma_{2} \gamma_{3} \approx \gamma_{5}
$$

It should be clear that in the case of Majorana such an operation cannot exist since $\Gamma_{0}$ is positive definite and the equation has not negative energy solutions. The reason why the operation does not exist is that for all the irreducible UR's of the Lorentz group the operator $B_{3}(\xi)$ has a pole at $\xi=i \pi$. The same happens for all the irreducible infinite dimensional represntations where the infinitesimal boost generator, $N_{3}$, is a normal operator. As a consequence of this pole, the standard derivation of the CPT theorem, as for instance in the Streater and Wightman book [40] based on the analytical continuation of the Lorentz group, does not hold. However, for all the finite dimensional representations of the Lorentz group, the operator $B_{3}(i \pi)$ exists and the CPT theorem is valid. It turns out that also the spin-statistics theorem is based on the existence of such an operator $\left(B_{3}(i \pi)\right)$ [40]. Therefore also this theorem cannot be proved in these theories. Of course the possibility of arranging the theory in such a way that CPT and spin-statistics are satisfied is not excluded. 
To stress the previous point let me notice that, also if the wave equation has negative energy solutions, the CPT theorem can be violated. Consider, for instance, the following wave equation [41]:

$$
\left(p^{\mu} \gamma_{\mu}-M-\frac{1}{2} \sigma^{\mu v}\left[\Gamma_{\mu}, \Gamma_{v}\right]\right) \psi=0
$$

with $\psi$ transforming as the direct product of the representations Dirac $\otimes$ Majorana. The existence of negative energy solutions is guaranteed by the CPT transformation

$$
p^{\mu} \rightarrow-p^{\mu}, \quad \gamma_{\mu} \rightarrow-\gamma_{\mu}
$$

Notice that under this transformation $\Gamma_{\mu} \rightarrow \Gamma_{\mu}$. Consider now a vector field $\phi^{\mu}$ with definite properties of transformations under the the previous CPT operation. We may construct the two local couplings:

$$
\bar{\psi} \gamma_{\mu} \psi \phi^{\mu}, \quad \bar{\psi} \Gamma_{\mu} \psi \phi^{\mu}
$$

Clearly one of these two couplings is not CPT invariant.

\section{Conclusions}

Nowdays the UR's of the Lorentz group do not seem to have interesting physical applications (however, see the attempts reviewed in [42]). However, in the paper that we have reviewed here, Majorana shows all his mathematical strength and ingenuity. There are several interesting points raised up by this paper. One is the question of the choice of representations made by Majorana. They enjoy many properties, they are the only irreducible UR's for which it is possible to define a four-vector operator in the sense specified in Section 2. Furthermore they are parity invariant. These properties can be easily seen by means of the group theoretical analysis developed here, so the question is if Majorana had these notions, or he arrived to these representations by simple chance. Considering that applying the same considerations it is easy to understand also the Majorana formulation of the massive neutral particle it seems more incredible that all this derived by casual circumstances.

Also, quite interestingly, the work of Majorana shows clearly that the CPT theorem can be violated in a local relativistic theory. In fact, relativity and locality are not enough to ensure the validity of this theorem. A further hypothesis about the nature of the representations of the Lorentz group is necessary. In particular the CPT theorem is valid for any finite dimensional representation. Therefore the only way to get a reasonable theory for a mass spectrum must involve necessarily finite dimensional representations (unless to introduce space-like solutions, violation of CPT and/ or lack of the spin-statistics connection). This happens in string theory which involves an infinite number of finite dimensional representations. It is curious that in the last case there are, in principle, problems with the positivity of the states. As well known this problem can be avoided by choosing a particular value for the space-time dimensions. On the contrary, in the case of unitary representations this problem does not arise, the metrics in the space of the states is positive definite, but of course, as we have seen, the theory has many other difficulties. 


\section{References}

[1] E. Majorana, Nuovo Cimento 9 (1932) 335.

[2] P.A.M. Dirac, Proceedings of the Royal Society A117 (1928) 610.

[3] P.A.M. Dirac, Proceedings of the Royal Society A133 (1931) 610.

[4] C.D. Anderson, Science 76 (1932) 238.

[5] D.M. Fradkin, American Journal of Physics 34 (1966) 314.

[6] H. Weyl, Gruppentherie und Quantunmechanik, S. Hierzel Verlag, Leipzig (1928).

[7] E. Majorana, Il Nuovo Cimento, 14 (1937) 171.

[8] Gel'fand and Yaglom, JETP 18 (1948) 703, 1096, 1105.

[9] V.L. Ginzburg, Acta Physica Polonica 15 (1956) 163.

[10] I.M. Gel'fand, R.A. Minlos and Z. Ya. Shapiro, Representations of the Rotation and Lorentz Group and their Applications, Pergamon Press (1963).

[11] M. Naimark, Linear Representations of the Lorentz Group, Pergamon Press (1964).

[12] Physikalische Berchte (1933) - I 548.

[13] E. Wigner, Annals of Mathematics 40 (1939) 149.

[14] R. F. Streater, http://www.mth.kcl.ac.uk/ streater/rongspin.html

[15] R. F. Streater, Commun. in Mathematical Phys. 5 (1967) 88.

[16] F. Gursey and L. A. Radicati, Phys. Rev. Lett. 13 (1964) 173.

[17] B. Sakita, Phys. Rev. 136 (1964) B1756.

[18] B. Sakita, Phys. Rev. Lett. 13 (1964) 643.

[19] Y. Dothan, M. Gell-Mann and Y. Ne'eman, Physics Letters 17 (1965) 148.

[20] A.O. Barut and H. Kleinert, Physical Review 156 (1967) 1541.

[21] C. Fronsdal, Physical Review 156 (1967) 1665.

[22] P.A.M. Dirac, Proceedings of the Royal Society A183 (1945) 284.

[23] H. Yukawa, Physical Review 77 (1953) 219

[24] H. Yukawa, Phys. Rev. 91 (1953) 415.

[25] C. Fronsdal, Physical Review 156 (1967) 1665.

[26] Y. Nambu, Supplements of Progress in Theoretical Physics 37 and 38 (1966) 368.

[27] Y. Nambu, Physical Review 160 (1967) 1171.

[28] G. Feldman and P.T. Matthews, Physical Review 154 (1967) 1241.

[29] C. Fronsdal, Physical Review 154 (1967) 1241.

[30] R.F. Dashen and M. Gell-Mann, Algebra of current componets at infinite momentum, Proceedings $3^{r} d$ Coral Gables Conference on "Symmetry Principles at High Energy", W.H. Freeman Co., San Francisco (1966). 
[31] M. Gell-Mann, Current algebra. Strong and weak interactions, International School of Physics E. Majorana (1966).

[32] F. Coester and G. Roepstoff, Physical Review 155 (1967) 1583.

[33] R.F. Dashen and M. Gell-Mann, Physical Review Letters 17 (1966) 349.

[34] M. Gell-Mann, Recent work on representations of current algebra, International School of Physics E. Majorana (1967).

[35] M. Gell-Mann, D. Horn and J. Weyers, Proceedings of the Heidelberg Conference on High Energy Physics and Elementary particles, (1967).

[36] I.T. Grodsky, M. Martinis and M. Swiecki, Physical Review Letters 19 (1967) 332.

[37] R. Casalbuoni, R. Gatto and G. Longhi, Physical Review D3 (1971) 1499.

[38] I.T. Grodsky and R.F. Streater, Physical Review Letters 21 (1967) 695.

[39] R. Casalbuoni and G. Longhi, Il Nuovo Cimento 15A (1973) 591.

[40] R.F. Streater and A.S. Wightman, PCT, Spin and Statistics, and All That, W.A. banjamin and Co. (1964).

[41] E. Abers, I.T. Grodsky and R.E. Norton, Physical Review 159 (1967) 1222.

[42] M. S. Plyushchay, EJTP 3, No. 10 (2006) 17, arXiv:math-ph/0604022. 